\title{
Application of BI-RADS Descriptors in Contrast-Enhanced Dual-Energy Mammography: Comparison with MRI
}

\author{
Thomas Knogler $^{a, b}$ Peter Homolka ${ }^{c}$ Mathias Hoernig ${ }^{d}$ Robert Leithner ${ }^{c}$ Georg Langs ${ }^{a, d}$ \\ Martin Waitzbauer ${ }^{a, d}$ Katja Pinker ${ }^{a, b, f}$ Sabine Leitner ${ }^{a, b}$ Thomas H. Helbich ${ }^{a, b}$ \\ a Department of Biomedical Imaging and Image-guided Therapy, Medical University of Vienna, Vienna, Austria; \\ ${ }^{b}$ Division of Molecular and Gender Imaging, Medical University of Vienna, Vienna, Austria; \\ ${ }^{\mathrm{c}}$ Center for Medical Physics and Biomedical Engineering, Medical University of Vienna, Vienna, Austria; \\ ${ }^{\mathrm{d}}$ X-Ray Products, Healthcare, Siemens AG, Erlangen, Germany; \\ e Computational Imaging Research Lab, Medical University of Vienna, Vienna, Austria \\ f Department of Radiology, Breast Imaging Service, Memorial Sloan Kettering Cancer Center, New York, NY, USA
}

\section{Keywords}

Breast cancer · Magnetic resonance imaging ·

Mammography · Contrast agent

\section{Summary}

Background: Contrast-enhanced (CE) magnetic resonance imaging (MRI) BI-RADS descriptors are used in the evaluation of contrast-enhanced dual-energy mammography (CEDEM) images of mass lesions and are assumed to be applicable. Patients and Methods: Patients with suspicious mass lesions on mammography ( $\mathrm{BI}$ RADS 4 or 5) were included. CEDEM examinations were performed using a modified prototype unit. CE-MRI was performed using a high temporal and high spatial resolution imaging protocol. 2 blinded breast radiologists evaluated all images using criteria related to contrast enhancement intensity and morphology according to the BI-RADS lexicon (5th edition) in 2 sessions. Histopathology was used as the standard of reference. Results: 11 patients with 5 benign and 6 malignant index lesions were included. Enhancement characteristics were similar in the malignant cases. Enhancement of the benign lesions was moderate on CEDEM and strong on MRI. Discrepancies in the BI-RADS descriptors did not influence the final BI-RADS score. Overall, the BI-RADS assessment was almost identical in all cases. 1 malignant lesion was rated BI-RADS 4 with CEDEM and BI-RADS 5 with $\mathrm{MRI}$, and 1 benign was rated BI-RADS 2 and $\mathrm{BI}-$ RADS 1, respectively. Conclusion: MRI BI-RADS descriptors of contrast-enhancing lesions can be applied for the morphologic analysis of mass lesions on CEDEM.

(C) 2017 S. Karger GmbH, Freiburg

\section{Introduction}

Contrast-enhanced (CE) magnetic resonance imaging (MRI) of the breast has emerged as a promising imaging tool with a high sensitivity for cancer detection $[1,2]$. However, specificity is limited, leading to an increased rate of supplementary diagnostic work-up [3]. CE-MRI is based on the depiction of newly formed proliferating tumor vessels with fragmented leaky basement membranes, also referred to as tumor neoangiogenesis [4-8]. Nevertheless, access to CE-MRI is limited to larger metropolitan areas, and the procedure is expensive and time-consuming. In addition, patients with metallic implants, pacemakers, allergies to gadoliniumbased contrast agents, or severe claustrophobia are not suitable for CE-MRI. Contrast-enhanced dual-energy mammography (CEDEM) is an emerging new X-ray technique that is also based on the depiction of tumor neoangiogenesis [9]. When added to mammography, it has the potential to increase diagnostic accuracy $[10,11]$ and can facilitate the visualization of breast lesions [11, 12]. Accuracy, sensitivity, specificity, lesion detection rate, and assessment of tumor size are comparable to CE-MRI of the breast [13-17]. The BI-RADS lexicon provides descriptors and assessment categories that can be used to predict the likelihood of malignancy for contrast-enhancing lesions on CE-MRI $[18,19]$. Recent studies have used MRI imaging characteristics for the evaluation of CEDEM images $[9,10,13,14]$; however, there is no data on the direct applicability of the MRI BI-RADS descriptors for contrastenhancing lesions on CEDEM.

The purpose of this study was to evaluate the comparability of the BI-RADS descriptors of contrast-enhancing mass lesions on CEDEM and MRI, and to ascertain whether this approach results in the same BI-RADS score.

\section{KARGER}

() 2017 S. Karger GmbH, Freiburg
Thomas H. Helbich, MD 


\section{Patients and Methods}

This prospective study was approved by the institutional review board Written informed consent was obtained. The equipment was provided by Siemens Healthcare (Erlangen, Germany), and the authors had complete control of the data and the article submitted for publication. Some patients and imaging data were used in a previous study [20].

\section{Patients}

Study enrollment was offered to patients who were recalled for suspicious mass lesions on screening mammography (American College of Radiology (ACR) BI-RADS 4 or 5 according to the 5th edition of the BI-RADS lexicon). Exclusion criteria were breast implants, any history of breast surgery, or prior cancer treatment due to the unknown effect of implants and scars on the subtraction algorithm for the calculation of the CEDEM image. Further exclusion criteria were metallic implants, contraindications for the administration of iodine- or gadolinium-based contrast agents, and pregnancy. In premenopausal women, examinations were performed in the second week of the menstrual cycle.

\section{CEDEM}

All CEDEM examinations were performed using a modified Siemens Mammomat Inspiration unit (Siemens Healthcare). CEDEM was performed as described previously [20]. Briefly, an image pair, consisting of a high-energy (HE; above the k-edge of iodine, $33.2 \mathrm{keV}$ ) and a low-energy (LE; below the k-edge) image, was obtained consecutively during a single breast compression after contrast medium administration. Tube voltage for $\mathrm{HE}$ was fixed at $49 \mathrm{kVp}$ and varied between 26 and $32 \mathrm{kVp}$ for LE images dependent on breast thickness and glandularity. Breast positioning and compression force for CEDEM were equal to those of conventional mammography. Non-ionic iodine contrast medium (Iomeron ${ }^{\circledR} 400$, Bracco, Milan, Italy) at $2 \mathrm{ml} / \mathrm{kg}$ body weight and a rate of $3.5 \mathrm{ml} / \mathrm{s}$ was administered with a power injector (Ulrich Medical, Ulm, Germany), followed by a $25-\mathrm{ml}$ saline flush. After $60-90 \mathrm{~s}$, the breast was compressed and imaging began. In this prototype study, imaging was restricted to 1 view where the lesions were best visible, in order to clearly depict the anatomical details. However, in the clinical setting, 2 views are mandatory. $\mathrm{HE}$ and LE images were registered non-rigidly to compensate for possible subtle tissue motion. Subtracted CEDEM images were generated via weighted subtraction using a fully automatic, locally adjusted, tissue thickness-dependent subtraction factor to avoid over-subtraction at the breast border [20].

\section{Image Acquisition - MRI}

MRI examinations were performed on a 3 Tesla MR Scanner (Tim Trio ${ }^{\mathrm{TM}}$, Siemens Healthcare) with a dedicated 4-channel double breast coil. A combined high temporal and high spatial resolution MRI protocol was used [21]. All examinations were performed in prone position. Examinations were performed using $0.2 \mathrm{mmol} / \mathrm{kg}$ body weight Gd-DOTA (gadoterate meglumine, Dotarem ${ }^{\circledR}$, Guerbet, Villepinte, France) intravenous contrast agent, injected with a power injector at $4 \mathrm{ml} / \mathrm{s}$ followed by a 25-ml saline flush. MRI and CEDEM examinations were scheduled for the same day in premenopausal woman and within 14 days in postmenopausal woman. The examination order was depended on timetable availability. The time interval between CEDEM and MRI was greater than $1 \mathrm{~h}$ when scheduled on the same day.

\section{Image Evaluation}

All MRI scans and CEDEM images were evaluated independently by 2 senior breast radiologists with more than 6 years' experience in breast imaging. In the case of a discrepancy, the final decision was made in consensus. No information on inclusion criteria, clinical data, patient history, or previous mammograms was provided. Screening full field digital mammography (FFDM) images were also available in all patients.

Reading was performed in 2 independent sessions. First, the CEDEM images were reviewed. 30 days later, MR images were evaluated with the whole set of acquired sequences available. Images were anonymized and shown in random order. All images were reviewed based on the criteria from the BI-RADS lexicon [19]. CEDEM images were evaluated using criteria related to contrast enhancement intensity and morphology according to the MRI part of the BIRADS lexicon [19]. Subjective judgment of lesion enhancement was performed based on the scale of none, mild, moderate, and strong $[22,23]$. Lesion size measurements were rated equal when the difference between the greatest diameters was lower than $0.5 \mathrm{~cm}$ in the enhancing index lesions. The same criterion was used for the whole extent of the multicentric/multifocal lesions. The final diagnosis was established via core needle biopsy and/or surgery in all patients [24]. The histopathologic diagnosis was used as the standard of reference.

\section{Results}

A total of 11 female patients (median age 58 years; range $45-74$ years) with 5 benign and 6 malignant index lesions were included in this study over a 9-month period. The details for each patient are summarized in table 1 . There were no signs of allergic reactions after the administration of any of the contrast agents. Of the 6 patients with malignant lesions, 3 had a single lesion, 1 had a multifocal cancer with 4 lesions (patient 5; fig. 1), and 2 had a multicentric cancer with 3 and 5 lesions, respectively (patients 6 and 10; fig. 2). Breast density was ACR A in 1, ACR B in 6, ACR C in 2, and ACR $\mathrm{D}$ in 2 patients. Background parenchymal enhancement was minimal in 2, mild in 4, moderate in 4, and marked in 1 patient, and was symmetrical in all. Overall, the BI-RADS assessment was almost identical in all cases. 1 malignant lesion was rated BI-RADS 4 on CEDEM and BI-RADS 5 on MRI. 1 benign lesion was rated BIRADS 2 on CEDEM and BI-RADS 1 on MRI. An inflammatory stromal change (patient 7; fig. 3) showed signs of malignancy and was rated BI-RADS 5 on both imaging tests, and thus resulted in 1 false-positive case. Enhancement characteristics were similar in both imaging tests. Strong enhancement was usually seen in malignant lesions. The enhancement of the 2 enhancing benign lesions was moderate on CEDEM and strong on MRI. Regarding the BIRADS descriptors, discrepancies were noted with respect to shape in 1 cancer and with respect to margin in 1 benign and 1 malignant lesion. However, the final BI-RADS score was not influenced. Both imaging tests depicted the same number of additional lesions. Those were small contrast-enhancing lesions with a diameter of up to $1 \mathrm{~cm}$ and imaging characteristics comparable to the index lesions, and proved to be malignant in the final histopathologic work-up. Size measurements were comparable in all enhancing index lesions. The size measurement in the multifocal/multicentric cases was almost identical between the 2 techniques.

\section{Discussion}

Our study shows that the BI-RADS descriptors for CE-MRI can be safely applied for CEDEM. Discrepancies were rare and did not influence the final BI-RADS score. Thus, MRI BI-RADS descriptors can be applied for the analysis of mass lesions in CEDEM.

We saw a high agreement rate between CEDEM and MRI for the BI-RADS descriptors shape, margin, lesion enhancement, and internal enhancement in malignant as well as in benign lesions. In 


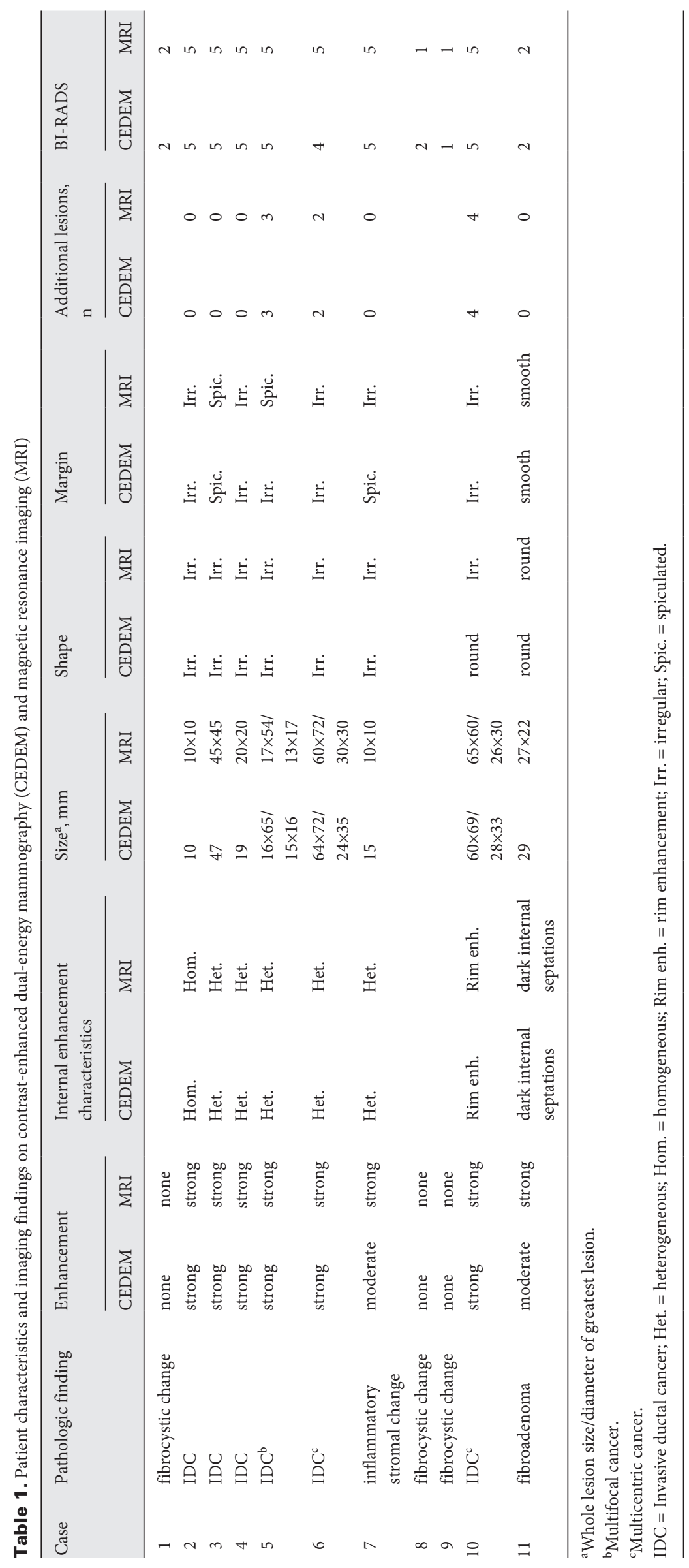


Fig. 1. Contrastenhanced dual-energy mammography (CEDEM) image in a cranio-caudal view, b subtracted paraxial multiplanar reformat $\mathrm{T} 1$ contrast-enhanced (CE) MRI image, c magnified CEDEM image in cranio-caudal view, and d magnified subtracted paraxial multiplanar reformat T1 CE-MRI from a 68-year-old female with a multifocal invasive ductal carcinoma. Comparable contrast enhancement, margins, and shape in the biggest lesion (arrow in $c, d)$, as well as in the ad-
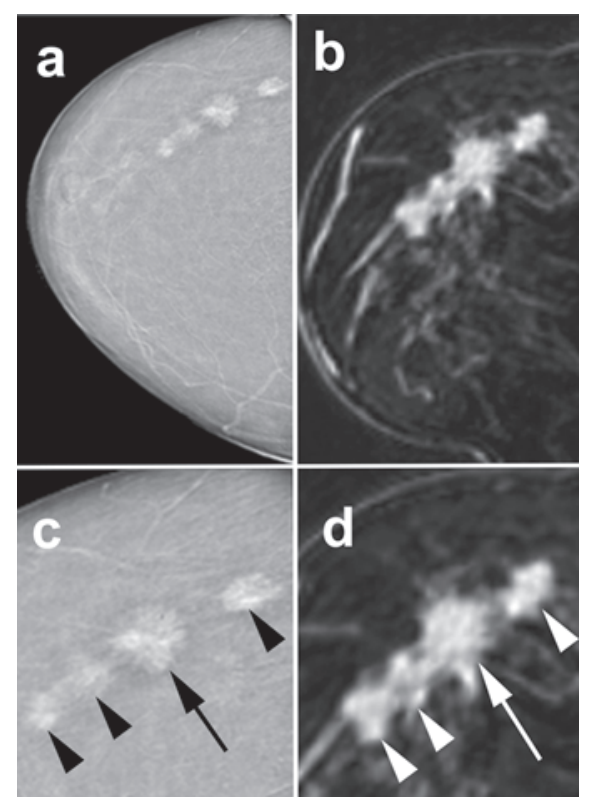

ditional lesions (arrowheads in c,d) are shown in both techniques.

Fig. 2. Contrastenhanced dual-energy mammography (CEDEM) image in a cranio-caudal view, b subtracted axial $\mathrm{T} 1$ contrast-enhanced (CE) MRI image, c magnified CEDEM image in cranio-caudal view, and d magnified subtracted axial T1 CE-MRI image from a 45-year-old female with a multicentric invasive ductal carcinoma. The rim enhance ment of the cancers is
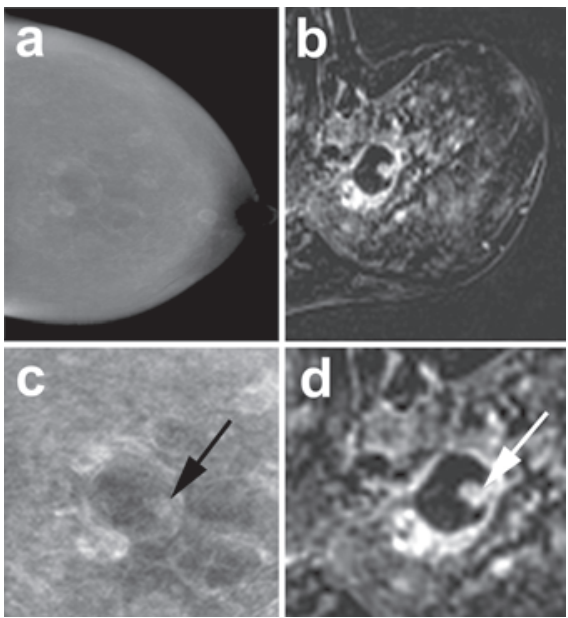

depicted equally on CEDEM (a,c) and CE-MRI (b,d). A contrast-enhancing nodule is also well depicted in CEDEM (arrow in c,d).

Fig. 3. Contrast-enhanced dual-energy mammography (CEDEM) image in a cranio-caudal view, b subtracted axial $\mathrm{T} 1$ contrast-enhanced (CE)-MRI image, c magnified CEDEM image in cranio-caudal view, and $\mathbf{d}$ magnified subtracted axial T1 CEMRI image from a

74-year-old female with an inflammatory stromal change (arrow in c,d). Although there were
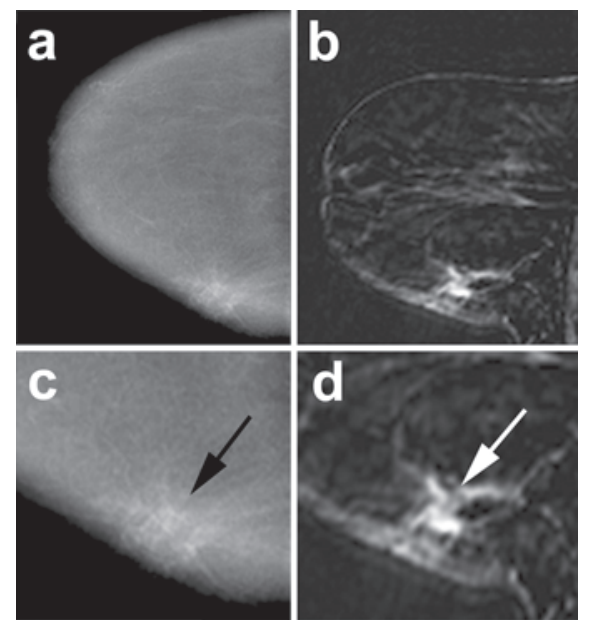

slight discrepancies in the interpretation of the enhancement and margins, the final BI-RADS grading was equal on CEDEM and CE-MRI.
2 malignant tumors, the margin descriptors, irregular and spiculated, were used differently. However, both are features with the highest predictive value for malignancy [18]. In another malignant case, the lesion shape was rated round on CEDEM and irregular on MRI. An irregular shape is more likely to be malignant; however, a round shape can also be associated with malignancy [18]. Nevertheless, this lesion was still rated as highly suggestive of malignancy due to the irregular margins, the strong contrast enhancement, and the rim enhancement, and did not lead to a missed cancer case on CEDEM. In accordance with other studies, strong contrast enhancement on CEDEM was highly suggestive for malignancy [9, 10]. Moreover, we can confirm that the enhancement characteristics in malignant lesions were equal on CEDEM and MRI $[13,15]$. We can also support the findings of Jong et al. [25] that enhancing benign lesions can be depicted with CEDEM. The enhancement was moderate on CEDEM and strong on MRI in both enhancing benign lesions. This observation is in accordance with Łuczyńska et al. [23] who reported that benign lesions most frequently show medium or weak enhancement on CE mammography. However, there is no data on the different enhancement of benign lesions on CEDEM and MRI. The lesion shape was rated equally. 1 benign case was false-positive in both techniques. Here, as in the true-positive cases, irregular and spiculated were used differently. However, this slight difference did not influence the final classification (fig. 3). Furthermore, CEDEM was able to depict dark internal septations in all fibroadenomas. The septations correlate with collagenous bands in histopathologic analysis and have a high predictive value for benign breast lesions [19].

Although there were some differences in single BI-RADS descriptors, the overall BI-RADS score was equal in all except 2 cases. However, there would have been no change in the clinical work-up according to CEDEM.

Compared to other studies on CE mammography, we were able to perform a detailed morphologic analysis of all lesions according to the BI-RADS lexicon, and compared these findings to the MRI descriptors. 1 false-positive case was identical with both techniques. This suggests a limited specificity of CEDEM comparable to that of MRI [26]. However, the real specificity of CEDEM is still unclear. Further larger studies are necessary.

In line with Jochelson et al. [15] and Fallenberg et al. [13], we were able to detect additional lesions with high sensitivity. There was an accurate correlation of the lesion size on CEDEM and on MRI in the index lesions as well as in the whole tumor extent in multifocal and multicentric cancers (figs. 1 and 2).

The data from our prototype study is limited due to the small sample size and the unbalanced distribution of malignant and benign cases.

Due to the prototype setting, the number of patients included in the study was low. Certain differences in interpretation between the 2 methods cannot be excluded. The different kinetics of iodineand gadolinium-based contrast agents might influence lesion morphology. This could lead to a different interpretation of BI-RADS descriptors on CEDEM and MRI. Thus, studies with larger patient numbers are needed to overcome this limitation. 
Nevertheless, the analyzed cases demonstrated that MRI BIRADS descriptors of contrast-enhancing lesions can be applied for the morphologic analysis of mass lesions on CEDEM. In addition, we only included mass lesions. However, we believe that the MRI BI-RADS descriptors for non-mass enhancing lesions are applicable for CEDEM as well. Nevertheless, this should be evaluated in a separate study. In addition, due to the prototype setting, we did not assess the contrast kinetics of the lesions, as the feasibility had already been demonstrated by Dromain et al. [9].

In conclusion, the preliminary results from our small sample size study show that the MRI BI-RADS descriptors of contrast-enhancing lesions can be applied for the morphologic analysis of mass lesions on CEDEM. Partial discrepancies in the interpretation did not influence the final BI-RADS score. A good differentiation between benign and malignant lesions is possible with the application of the MRI BI-RADS descriptors in CEDEM. However, studies with larger patient numbers, including non-mass lesions and contrast kinetics, are necessary.

\section{Disclosure Statement}

This study has received funding by Siemens Healthcare, Erlangen, Germany and Bracco, Milan, Italy. M.H. is an employee of Siemens Healtcare, Erlangen, Germany.

\section{References}

1 Heywang-Kobrunner SH, Hacker A, Sedlacek S: Magnetic resonance imaging: the evolution of breast imaging. Breast 2013;22(suppl 2):S77-82.

2 Weinstein S, Rosen M: Breast MR imaging: current in dications and advanced imaging techniques. Radiol Clin North Am 2010;48:1013-1042.

3 Menezes GL, Knuttel FM, Stehouwer BL, Pijnappel $\mathrm{RM}$, van den Bosch MA: Magnetic resonance imaging in breast cancer: a literature review and future perspectives. World J Clin Oncol 2014;5:61-70.

4 Folkman J: What is the evidence that tumors are angiogenesis dependent? J Natl Cancer Inst 1990;82:4-6.

5 Nagy JA, Brown LF, Senger DR, Lanir N, van de Water L, Dvorak AM, Dvorak HF: Pathogenesis of tumor stroma generation: a critical role for leaky blood vessels and fibrin deposition. Biochim Biophys Acta 1989; 948:305-326.

6 Weidner N, Semple JP, Welch WR, Folkman J: Tumor angiogenesis and metastasis-correlation in invasive breast carcinoma. N Engl J Med 1991;324:1-8.

7 Weidner N, Folkman J, Pozza F, Bevilacqua P, Allred EN, Moore DH, Meli S, Gasparini G: Tumor angiogenesis: a new significant and independent prognostic indicator in early-stage breast carcinoma. J Natl Cancer Inst 1992;84:1875-1887.

8 Buadu LD, Murakami J, Murayama S, Hashiguchi N Sakai S, Masuda K, Toyoshima S, Kuroki S, Ohno S: Breast lesions: correlation of contrast medium enhancement patterns on MR images with histopathologic findings and tumor angiogenesis. Radiology 1996;200:639-649.

9 Dromain C, Balleyguier C, Muller S, Mathieu MC Rochard F, Opolon P, Sigal R: Evaluation of tumor angiogenesis of breast carcinoma using contrastenhanced digital mammography. AJR Am J Roentgenol 2006; 187:W528-537.

10 Diekmann F, Freyer M, Diekmann S, Fallenberg EM, Fischer T, Bick U, Pollinger A: Evaluation of contrastenhanced digital mammography. Eur J Radiol 2011;78: 112-121.
11 Mori M, Akashi-Tanaka S, Suzuki S, Daniels MI, Watanabe C, Hirose M, Nakamura S: Diagnostic accuracy of contrast-enhanced spectral mammography in comparison to conventional full-field digital mammography in a population of women with dense breasts. Breast Cancer 2017;24:104-110.

12 Dromain C, Thibault F, Diekmann F, Fallenberg EM, Jong RA, Koomen M, Hendrick RE, Tardivon A, Toledano A: Dual-energy contrast-enhanced digital mammography: initial clinical results of a multireader, multicase study. Breast Cancer Res 2012;14:R94.

13 Fallenberg EM, Dromain C, Diekmann F, Engelken F, Krohn M, Singh JM, Ingold-Heppner B, Winzer KJ, Bick U, Renz DM: Contrast-enhanced spectral mammography versus MRI: initial results in the detection of breast cancer and assessment of tumour size. Eur Radiol 2014;24:256-264.

14 Lobbes MB, Lalji UC, Nelemans PJ, Houben I, Smidt ML, Heuts E, de Vries B, Wildberger JE, Beets-Tan RG: The quality of tumor size assessment by contrastenhanced spectral mammography and the benefit of additional breast MRI. J Cancer 2015;6:144-150.

15 Jochelson MS, Dershaw DD, Sung JS, Heerdt AS, Thornton C, Moskowitz CS, Ferrara J, Morris EA: Bilateral contrast-enhanced dual-energy digital mammography: feasibility and comparison with conventional digital mammography and MR imaging in women with known breast carcinoma. Radiology 2013; 266:743-751.

16 Chou CP, Lewin JM, Chiang CL, Hung BH, Yang TL, Huang JS, Liao JB, Pan HB: Clinical evaluation of contrast-enhanced digital mammography and contrast enhanced tomosynthesis - comparison to contrast-enhanced breast MRI. Eur J Radiol 2015;84:2501-2508.

17 Fallenberg EM, Schmitzberger FF, Amer H, IngoldHeppner B, Balleyguier C, Diekmann F, Engelken F, Mann RM, Renz DM, Bick U, Hamm B, Dromain C: Contrast-enhanced spectral mammography vs. mammography and MRI - clinical performance in a multireader evaluation. Eur Radiol 2017;27:2752-2764.

18 Mahoney MC, Gatsonis C, Hanna L, DeMartini WB, Lehman C: Positive predictive value of BI-RADS MR imaging. Radiology 2012;264:51-58.
19 D’Orsi CJ, Sickles EA, Mendelson EB, Morris EA: ACR BI-RADS Atlas, Breast Imaging Reporting and Data System. Reston, VA, American College of Radiology, 2013

20 Knogler T, Homolka P, Hornig M, Leithner R, Langs G Waitzbauer M, Pinker-Domenig K, Leitner S, Helbich $\mathrm{TH}$ : Contrast-enhanced dual energy mammography with a novel anode/filter combination and artifact reduction: a feasibility study. Eur Radiol 2016;26:15751581.

21 Pinker K, Grabner G, Bogner W, Gruber S, Szomolanyi P, Trattnig S, Heinz-Peer G, Weber M, Fitzal F, Pluschnig U, Rudas M, Helbich T: A combined high temporal and high spatial resolution 3 Tesla MR imaging protocol for the assessment of breast lesions: initial results. Invest Radiol 2009;44:553-558.

22 Lewin JM, Isaacs PK, Vance V, Larke FJ: Dual-energy contrast-enhanced digital subtraction mammography: feasibility. Radiology 2003;229:261-268.

23 Luczynska E, Niemiec J, Hendrick E, Heinze S, Jaszczynski J, Jakubowicz J, Sas-Korczynska B, Rys J: Degree of enhancement on contrast enhanced spectral mammography (CESM) and lesion type on mammography (MG): comparison based on histological results. Med Sci Monit 2016;22:3886-3893.

24 Wallis M, Tardivon A, Helbich T, Schreer I; European Society of Breast Imaging: Guidelines from the European Society of Breast Imaging for diagnostic interventional breast procedures. Eur Radiol 2007;17:581-588.

25 Jong RA, Yaffe MJ, Skarpathiotakis M, Shumak RS, Danjoux NM, Gunesekara A, Plewes DB: Contrast-enhanced digital mammography: initial clinical experience. Radiology 2003;228:842-850.

26 Sardanelli F, Boetes C, Borisch B, Decker T, Federico M, Gilbert FJ, Helbich T, Heywang-Kobrunner SH, Kaiser WA, Kerin MJ, Mansel RE, Marotti L, Martincich L, Mauriac L, Meijers-Heijboer H, Orecchia R, Panizza P, Ponti A, Purushotham AD, Regitnig P, Del Turco MR, Thibault F, Wilson R: Magnetic resonance imaging of the breast: recommendations from the EUSOMA working group. Eur J Cancer 2010;46:12961316 